\title{
Research Article \\ Effect of Nitrogen Impurity on Electronic Properties of Boron Nanotubes
}

\author{
Sandeep Kumar Jain and Pankaj Srivastava \\ Nanomaterials Research Group, Computational Nanoscience \& Technology Lab, ABV-Indian Institute of \\ Information Technology \& Management, Morena Link Road, Gwalior 474015, India \\ Correspondence should be addressed to Pankaj Srivastava; pankajs@iiitm.ac.in
}

Received 17 May 2013; Accepted 25 November 2013; Published 6 February 2014

Academic Editor: Dario Alfe

Copyright (C) 2014 S. K. Jain and P. Srivastava. This is an open access article distributed under the Creative Commons Attribution License, which permits unrestricted use, distribution, and reproduction in any medium, provided the original work is properly cited.

For the first time we present electronic band structure and density of states for nitrogen doped hexagonal ultrathin boron nanotubes in the framework of density functional theory. The considered models of nanotubes below $5 \AA$ diameter are armchair $(3,3)$, zigzag $(5,0)$, and chiral $(4,2)$. The impurity chosen for the study is nitrogen and concentration of impurity atoms is limited to two. The study reveals that $(3,3)$ BNT retains its metallic nature after nitrogen doping. However, metallicity gets increased which is attributed by the excess electrons of nitrogen. Further, it also brings out that $(5,0)$ BNT which is originally metal transforms into semiconductor after nitrogen interaction and the band gap at G point increases with the impurity. Moreover, the band gap of $(4,2)$ BNT reduces significantly and turns into semimetal for nitrogen doping. Thus, the nitrogen impurity has the predominant effect on the electronic properties of BNTs and therefore can be regarded as suitable candidates for nanoelectronic and field emission devices.

\section{Introduction}

The realization of carbon nanotubes (CNTs) has fascinated the vast community of researchers because of their intriguing physical and chemical properties [1]. The CNTs were found to be either metallic or semiconducting depending on the chiral vectors $(n, m)$ of the lattice [2-4] and showing good electrochemical properties [5-8]. Therefore, many material scientists were urged to shed light on the applications of CNTs in electronic devices such as field effect transistors and field emitters for which tailoring and control of electronic properties become extremely critical $[9,10]$. The main problem with CNTs is the inability to control the chirality which has not been resolved since their discovery. However, these problems can be resolved by using another material, that is, boron. The boron nanotube (BNT) was first proposed by Boustani et al. [11, 12] and subsequently Ciuparu et al. [13] synthesized the pure BNT of diameter 3 nanometer $(\mathrm{nm})$ on Mg-MCM-41 catalyst that confirmed the existence of BNTs. The synthesized BNTs were found to be metallic irrespective of chirality. It is considered that BNTs are formed by rolling up the boron sheets (BS). Unlike the hexagonal graphene sheet, the boron sheets have various conformations, namely, hexagonal, triangular, and hybrid hexagonal-triangular $(\alpha-$ sheet). In addition, $\alpha$-boron sheet was found to be the most stable followed by triangular and hexagonal BS [14]. The $\alpha$ BNTs $[15,16]$ were predicted to be metallic for diameter $\geq 17 \AA$ and semiconducting for diameter $<17 \AA$. Moreover, armchair $(3,3)$ and zigzag $(5,0) \alpha$-BNTs were found to be semiconducting with small band gap and transformed into metallic when axial strain is applied [17]. Similarly, triangular BNTs are found to be metallic irrespective of diameter and chiral angles [18]. The CNT-like BNT has been proposed by Zhang et al. [19] and Jain and Srivastava [20] independently and they stated that BNTs are not limited to hexagonal pyramidal structures constructed from the so-called Aufbau principle, and, alternatively, it is proposed that the thinnest BNT may be a geometrical analog of the corresponding CNT. Crystalline BNTs were synthesized first time by thermal evaporation method [21] and revealed the perfect single crystal having tetragonal structure with their growth direction along $\left(\begin{array}{lll}0 & 0 & 1\end{array}\right)$. Further, it was investigated that BNTs are more conducting than those of CNTs $[22,23]$. The stability of BNTs changes significantly for small diameter and observed the most stable one of diameter $0.39 \mathrm{~nm}$ with distorted hexagonal structure [23]. 
The chemical doping of nanostructured materials is a possible route towards controllable modification of electronic properties and enhancing stability. Therefore, doping may be implemented by intercalating foreign atoms into the open space or by substituting the host atoms. The first synthesis of nitrogen doped CNT was reported by Glarup et al. [24] using arc discharge method. However, direct growth of nitrogen doped single wall carbon nanotubes (N-SWCNTs) by chemical vapour deposition (CVD) could be a preferable growth method for the device fabrication. Most of the nanotubes grown by CVD were multiwalled with bamboo shapes [25-27]. Recently, Villalpando-Paez et al. [28] synthesized nitrogen doped-SWCNTs at $950^{\circ} \mathrm{C}$ by CVD using a solution of ferrocene, ethanol, and benzylamine. However, the growth temperature is too high for the method to be adopted for device fabrication. In addition, the N-SWCNTs have been synthesized directly on $\mathrm{SiO}_{2} / \mathrm{Si}$ substrate at $450^{\circ} \mathrm{C}$ by water plasma chemical vapor deposition and have diameter in the range of $0.8-1.6 \mathrm{~nm}$ whereas the imperfection is decreased with the increase of nitrogen concentration [29]. The dopant nitrogen atom can be displaced more easily than the host carbon atoms [30]. Moreover, the enhancement of density of states (DOS) at Fermi level for N-doped CNTs reflects higher conductivity [31-33].

Therefore, the above N-doped experimental and theoretical investigations instigate us to explore the effect of $\mathrm{N}$ impurity on boron nanotubes (BNTs). The structural stability of N-doped BNTs has already been studied in our earlier work [34] and found energetically more stable as compared to pristine counterparts. It is our natural choice to use nitrogen impurity for present investigations as it has the size almost equal to boron atom. In this study, we have taken three morphologies of hexagonal SWBNTs, namely, armchair $(3,3)$, zigzag $(5,0)$, and chiral $(4,2)$, consisting of 12,20 , and 56 atoms, respectively. The current investigation deals with the ground state energy of relaxed structures of N-doped BNTs and thereby electronic band structures and density of states.

\section{Computational Methodology}

The pseudopotential plane wave calculations have been performed for determining electronic properties of small diameter BNTs. The nitrogen atoms substitute the host boron atoms in the middle of BNTs with the doping concentration limited to two atoms. The positions of substituted atoms are illustrated in Figures 1 and 2, respectively, for one nitrogen atom (N1) and two nitrogen atoms (N2) substituted BNTs. $\mathrm{OA}$ and $\mathrm{OB}$ represent the cross section while $\mathrm{OC}$ is along axis of the tube and periodic along axis. The diameters of these tubes are taken as $4.60 \AA, 4.78 \AA$, and $4.87 \AA$ for $(5,0)$, $(3,3)$, and $(4,2)$, respectively. However, it is expected that the diameter might change after optimization. The total energy of ground state is calculated by DFT based CASTEP [35] simulation tool. The plane wave cut-off energy is set to be $320 \mathrm{eV}$. The exchange correlation effects are described by generalized gradient approximation (GGA) [36] proposed by PerdewBurke-Ernzerhof (PBE). The integration is performed in the first Brillouin zone [37] by using the k-points generated by

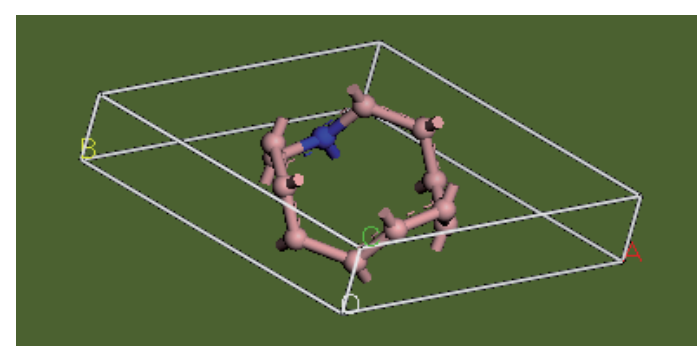

(a)

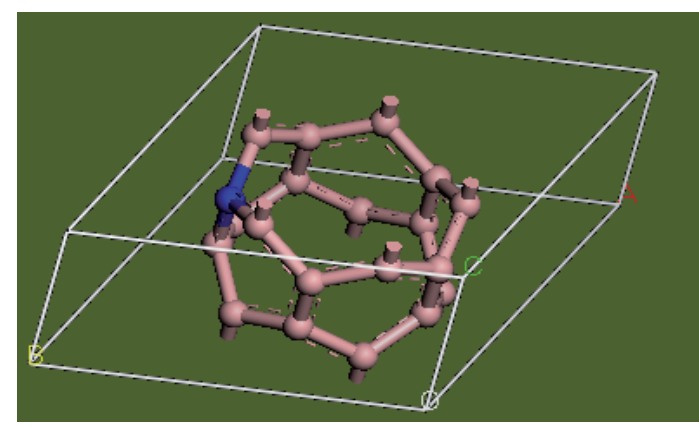

(b)

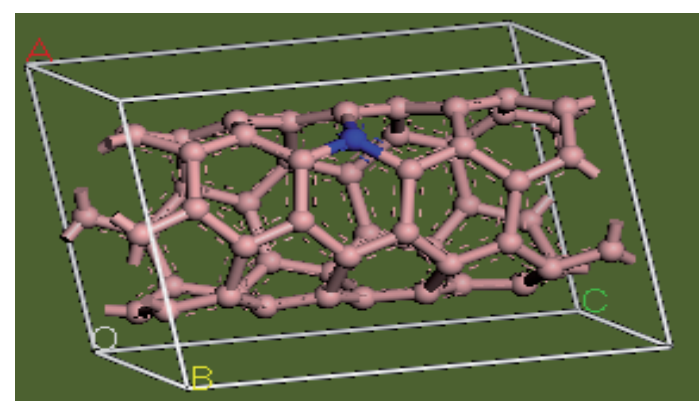

(c)

Figure 1: The ball and stick model of single atom of nitrogen doped for (a) armchair (3,3), (b) zigzag $(5,0)$, and (c) chiral boron nanotubes. The encircled blue atom is of nitrogen while brown is of boron.

$1 \times 1 \times 8$ grid parameters. The ultrasoft pseudopotentials are used in the reciprocal space [38] for optimizing the geometry. However, the structures are optimized until the force on each atom becomes smaller than $0.03 \mathrm{eV} / \AA$. The intertubular distance is set to $10 \AA$ in order to avoid periodic image interaction. The B-B bond length is chosen to be $1.67 \AA$ before optimization. Moreover, the considered calculations are non-spin-polarized.

\section{Results and Discussion}

The electronic band structures of N-doped hexagonal ultrathin boron nanotubes have been calculated and compared with pristine BNTs [20], CNTs [39], boron nitride nanotubes (BNNTs) [42], and $\mathrm{SiC}$ nanotubes [40] of same chiral vectors and summarized in Table 1 . It is evident from Table 1 that zigzag $(5,0)$ and armchair $(3,3)$ CNTs are metallic while chiral $(4,2)$ is semiconducting with narrow band gap. Similarly, 
TABLE 1: Comparison of electronic properties of various nanotubes.

\begin{tabular}{lllllll}
\hline Nanotubes & CNT $^{\mathrm{a}}$ & BNNT & SiCNT & BNT $^{\mathrm{c}}$ & $\begin{array}{l}\text { One N-doped } \\
\text { BNT }^{\mathrm{d}}\end{array}$ & $\begin{array}{l}\text { Two N-doped } \\
\text { BNT }^{\mathrm{d}}\end{array}$ \\
\hline Armchair $(3,3)$ & Metallic & $\begin{array}{l}\text { Semiconducting } \\
(6.06 \mathrm{eV})^{\mathrm{e}}\end{array}$ & $\begin{array}{l}\text { Semiconducting } \\
(2.13 \mathrm{eV})\end{array}$ & Metallic & Metallic & Metallic \\
\hline Zigzag $(5,0)$ & Metallic & $\begin{array}{l}\text { Semiconducting } \\
(2.1 \mathrm{eV})^{\mathrm{f}}\end{array}$ & $\begin{array}{l}\text { Semiconducting } \\
(0.19 \mathrm{eV})\end{array}$ & Metallic & $\begin{array}{l}\text { Semiconducting } \\
(1.11 \mathrm{eV})\end{array}$ & $\begin{array}{l}\text { Semiconducting } \\
(1.19 \mathrm{eV})\end{array}$ \\
\hline Chiral $(4,2)$ & $\begin{array}{l}\text { Semiconducting } \\
(0.26 \mathrm{eV})\end{array}$ & $\begin{array}{l}\text { Semiconducting } \\
(3.23 \mathrm{eV})^{\mathrm{f}}\end{array}$ & $\begin{array}{l}\text { Semiconducting } \\
(0.75 \mathrm{eV})\end{array}$ & $\begin{array}{l}\text { Semiconducting } \\
(0.43 \mathrm{eV})\end{array}$ & $\begin{array}{l}\text { Semimetallic } \\
(0.16 \mathrm{eV})\end{array}$ & $\begin{array}{l}\text { Semimetallic } \\
(0.0 \mathrm{eV})\end{array}$ \\
\hline
\end{tabular}

${ }^{a}$ Reference [39].

${ }^{\mathrm{b}}$ Reference [40].

${ }^{\mathrm{c}}$ Reference [20].

${ }^{\mathrm{d}}$ Present Study.

${ }^{\mathrm{e}}$ Reference [41].

${ }^{\mathrm{f}}$ Reference [42].

BNNTs and SiC nanotubes were found to be semiconducting with large band gaps. In addition, armchair $(3,3)$ and zigzag $(5,0)$ hexagonal ultrathin BNTs are metallic and chiral $(4,2)$ was found to be semiconducting with narrow band gap [20]. Besides, the electronic states are observed near the Fermi level giving high conductivity. The availability of the electronic states near the Fermi level increases with impurity which results in high conductivity. Furthermore, $2 \mathrm{p}$ electrons of $\mathrm{N}$ hybridize with the $2 \mathrm{p}$ electrons of $\mathrm{B}$; thereby the band structures get modified. It is also observed that the charge is transferred from boron (B) to nitrogen $(\mathrm{N})$ atom which may be the reason of additional states in the vicinity of Fermi level. These additional states are responsible for altering the properties. The nitrogen doping has predominant effects on electronic properties of nanotubes. Therefore, they are modified after nitrogen doping as illustrated in Table 1. The geometry of nanotubes remains cylindrical after $\mathrm{N}$ doping. However, the cross section of the nanotubes changes slightly. The band structures are plotted in the first Brillouin zone through the symmetric k-points G, F, Q, and $\mathrm{Z}$ which are corresponding to $(0.0,0.0,0.0),(0.0,0.5,0.0),(0.0,0.5,0.5)$, and $(0.0,0.0,0.5)$, respectively. Moreover, with a view to observe the effect of nitrogen impurity in the vicinity of Fermi level, the band structures and corresponding DOS are truncated to the energy range $-2 \mathrm{eV}$ to $2 \mathrm{eV}$. The detailed analysis of the nitrogen doping is provided in the subsequent sections.

The structural stability was predicted high for n-doped BNT in comparison to pristine ones [34]. Moreover, it was also predicted that the stability increases with impurity concentration. The chiral $(4,2)$ BNT was found to be the most stable followed by armchair $(3,3)$ and zigzag $(5,0)$. The same trend was also noticed in CNTs [39] and BNTs [42] of the same chirality. However, the difference in cohesive energies was very small which indicate that they are almost equally stable. Moreover, after nitrogen doping, the stability of BNTs becomes comparable to other BNTs formed from triangular, $\alpha$-, and hexagonal boron sheets [43].

3.1. Armchair $(3,3)$ Boron Nanotube. First of all we present the electronic band structure of single nitrogen atom (N1) doped BNT as depicted in Figure 3. It is evident that two

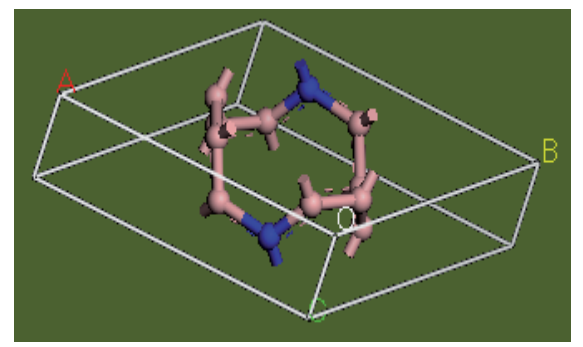

(a)

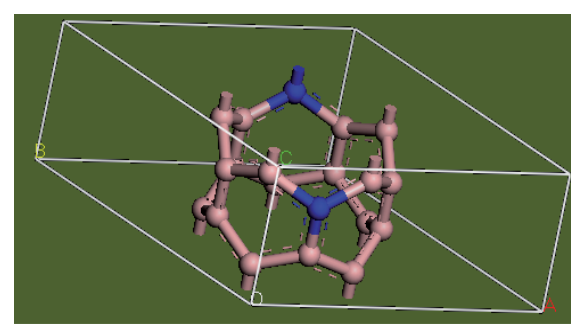

(b)

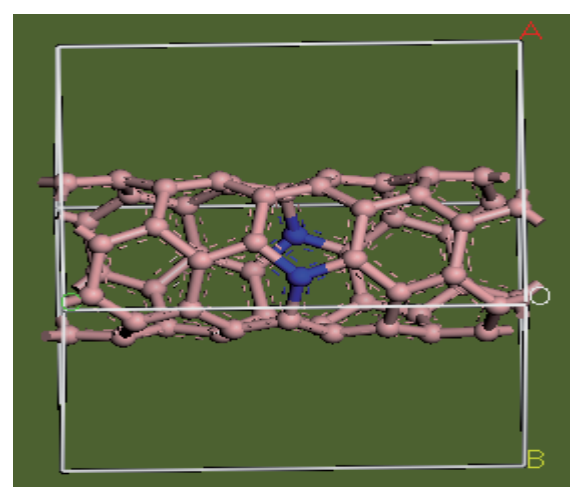

(c)

Figure 2: The ball and stick model of two atoms of nitrogen doped for (a) armchair (3,3), (b) zigzag (5,0), and (c) chiral boron nanotubes. The encircled blue atoms are of nitrogen while the brown are of boron.

electronic states cross the Fermi level indicating metallic nature. Moreover, the states are localized near the vicinity of Fermi level resulting in more availability of free electrons 


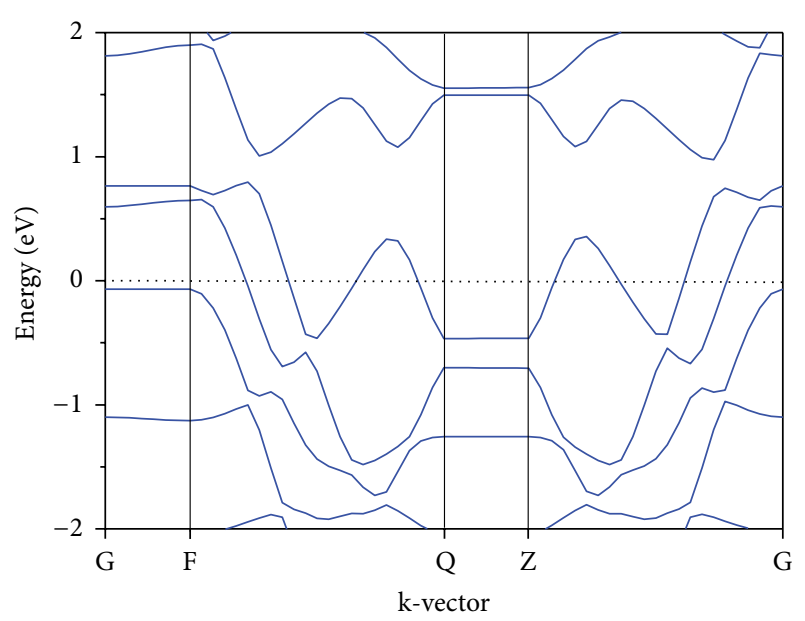

FIgURE 3: The electronic band structure of N1-doped single walled armchair $(3,3)$ BNT. The Fermi level is set corresponding to zero energy and represented by dotted line.

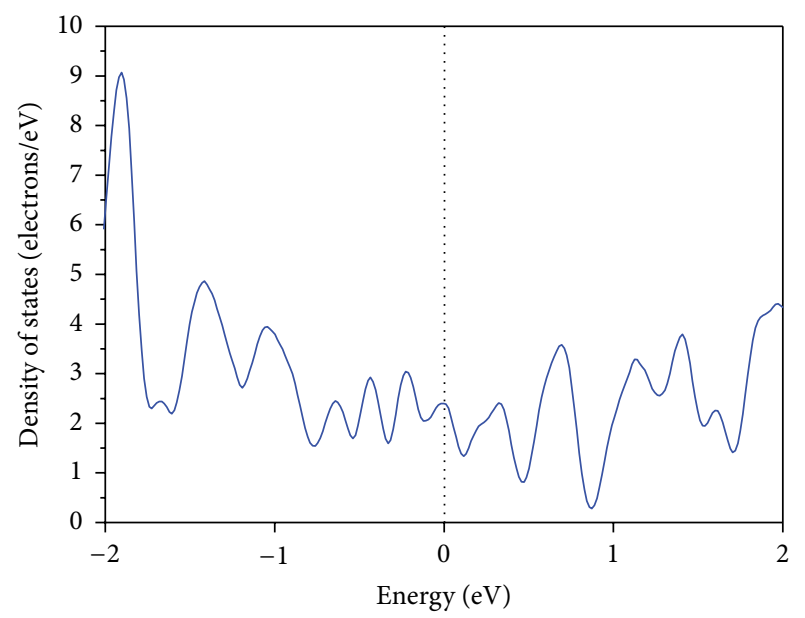

FIGURE 4: Density of states (DOS) of N1-doped single walled armchair $(3,3)$ boron nanotube. The Fermi energy is set corresponding to zero energy and represented by dotted line.

for conduction and extra energy state is produced by the additional electrons of $\mathrm{N}$ impurity. However, in our earlier work [20], we demonstrated that only one state crosses the Fermi level. In addition, the corresponding DOS is illustrated in Figure 4. The DOS profile shows a high peak at the Fermi level which ensures the metallic behavior of N1-doped $(3,3)$ BNT. The value of DOS at Fermi level is high compared to pristine one. Moreover, large numbers of free electrons are available in the vicinity of Fermi level. Here, peak of DOS is located at Fermi level which means valence band is filled. The high value of DOS at Fermi level represents high conductivity and chemical reactivity. Thus, the armchair $(3,3)$ BNT after N doping becomes more conducting as evidenced by Figures 3 and 4. It is also noticed that band structure and DOS profiles are in good agreement with each other.

Again, the concentration of impurity increases to two atoms, and the corresponding band structure and DOS are

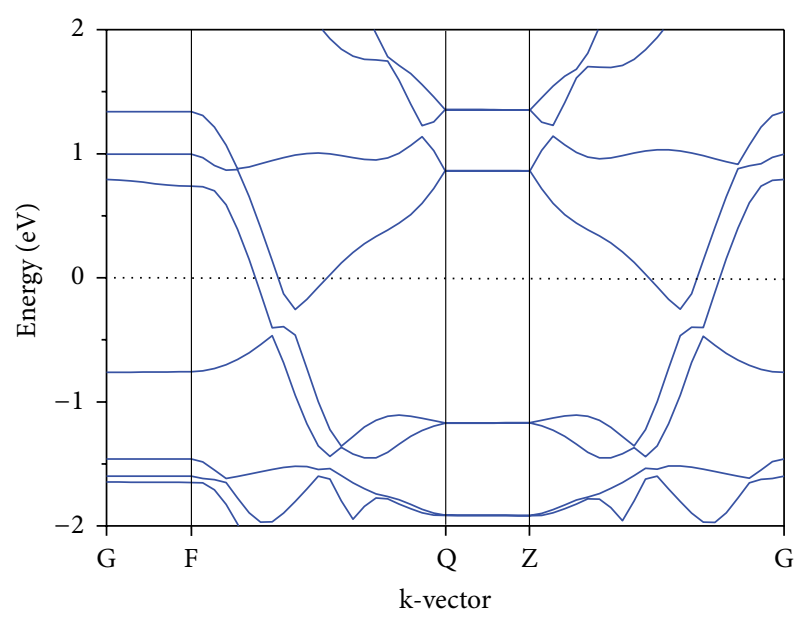

FIgURE 5: The electronic band structure of N2-doped single walled armchair $(3,3)$ BNT. The Fermi level is set corresponding to zero energy and represented by dotted line.

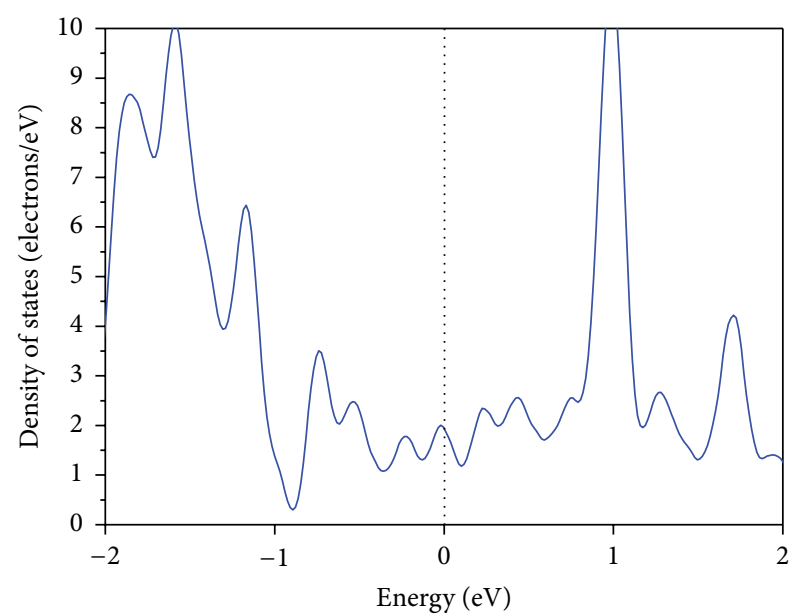

FIGURE 6: Density of states (DOS) of N2-doped single walled armchair $(3,3)$ boron nanotube. The Fermi energy is set corresponding to zero energy and represented by dotted line.

depicted in Figures 5 and 6, respectively. It is revealed that two energy levels cross the Fermi level which ensures metallic character as in N1-doped $(3,3)$ BNT. The energy states get dispersive near the Fermi level showing delocalized type nature but remain metallic. The DOS is shifted downward at Fermi level; thereby the conductivity and chemical reactivity reduced slightly. This effect may be caused due to the excess electrons of impurity atoms. Nevertheless, this favors the metallic nature of $\mathrm{N}$-doped armchair $(3,3)$ BNTs. Thus, armchair $(3,3)$ BNT still retains its original metallic nature even after $\mathrm{N}$ doping. Our findings are also consistent with CNT doped with N impurity. It is worthwhile to mention here that pristine $(3,3)$ BNT and CNT are found to be metallic while BNNT and SiCNT are wide band gap semiconductors. Thus, N-doped $(3,3)$ BNT is predicted to be highly metallic and therefore can be used as metallic contacts in nanodevices. 


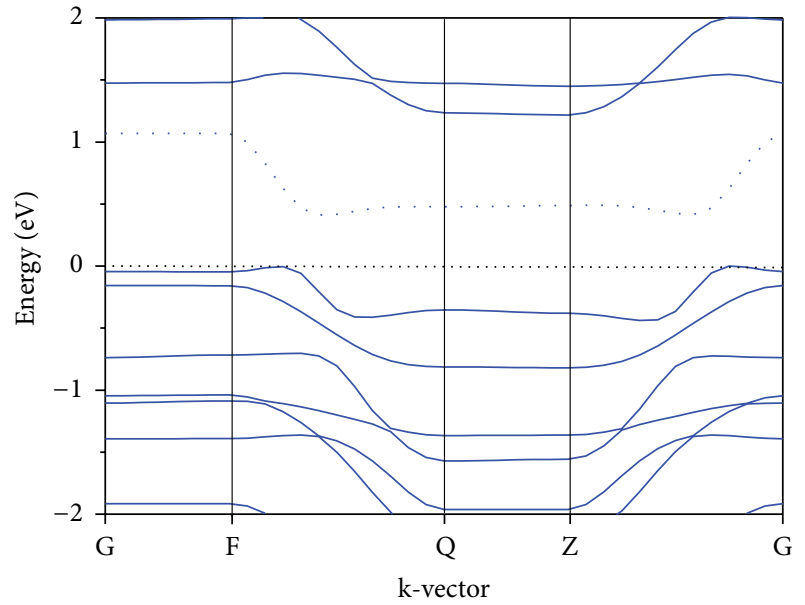

Figure 7: The electronic band structure of N1-doped single walled zigzag $(5,0)$ BNT. The Fermi level is set corresponding to zero energy and represented by dotted line.

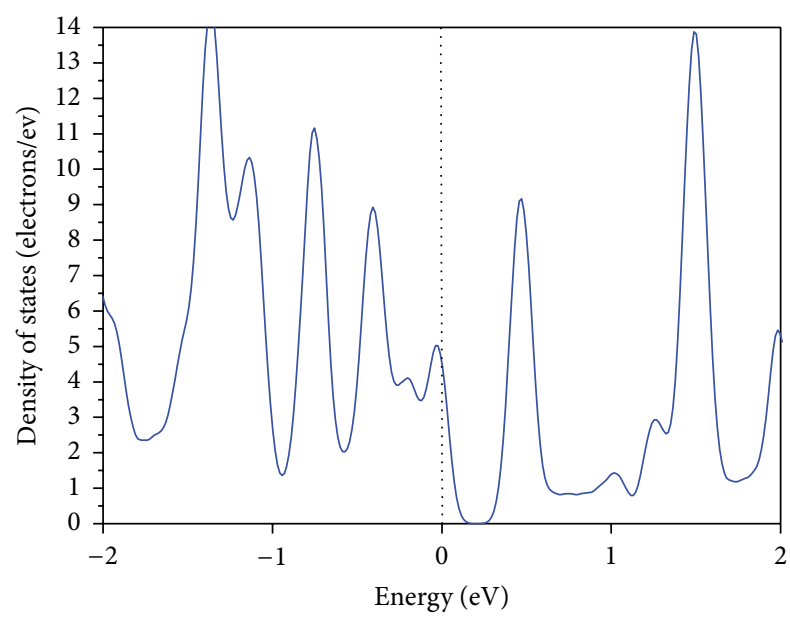

Figure 8: Density of states (DOS) of N1-doped single walled zigzag $(5,0) \mathrm{BNT}$. The Fermi energy is set corresponding to zero energy and represented by dotted line.

3.2. Zigzag $(5,0)$ Boron Nanotube. In this section, we present electronic properties of N-doped zigzag $(5,0)$ BNTs. The band structure of N1-doped BNT is shown in Figure 7. The band gaps have been examined at different symmetric k-points and found to be semiconducting. The band gap at the most symmetric point $\mathrm{G}$ is observed to be $1.11 \mathrm{eV}$ but $0.84 \mathrm{eV}$ at another symmetric point $\mathrm{Z}$. However, an indirect band gap $0.41 \mathrm{eV}$ is noticed somewhere between $\mathrm{G}$ and $\mathrm{Z}$. The DOS for the same is depicted in Figure 8. One can notice a sharp peak near the Fermi level in the valence band (VB) which ensures that the VB is not filled. However, more electronic states are localized in the vicinity of the Fermi level and provide large free charge carriers.

In addition, the band structure of N2-doped $(5,0)$ BNT is demonstrated in Figure 9. As the content of $\mathrm{N}$ increases, the free electrons also increase thereby affecting the band structure. The modified band gap at the most symmetric

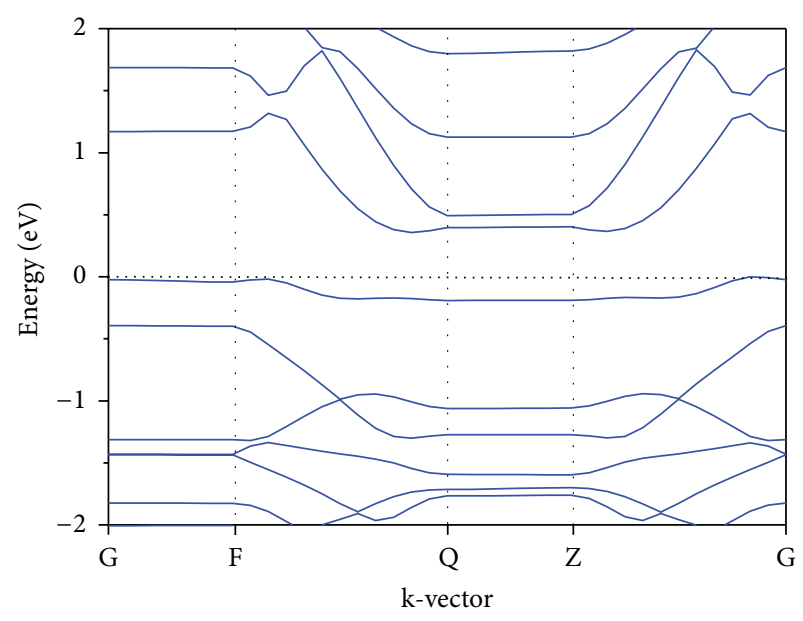

FIGURE 9: The electronic band structure of N2-doped single walled zigzag $(5,0)$ BNT. The Fermi level is set corresponding to zero energy and represented by dotted line.

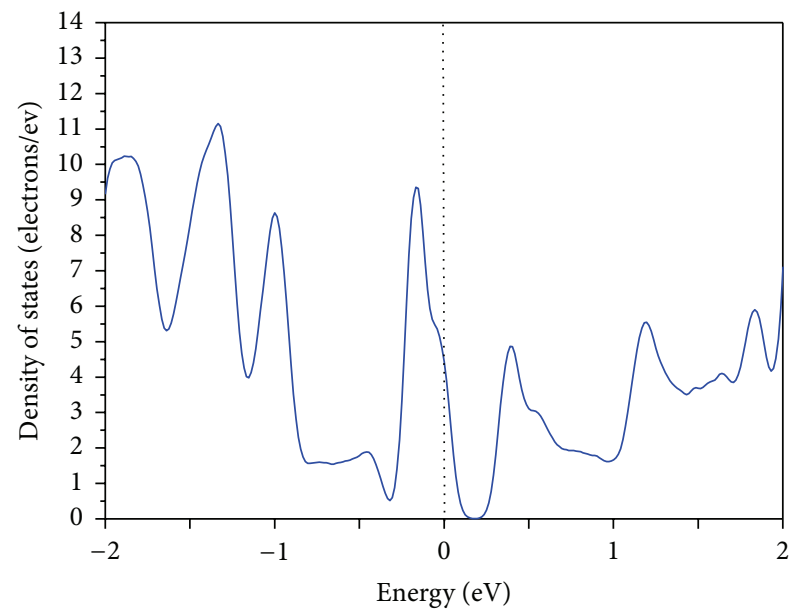

FIGURE 10: Density of states (DOS) of N2-doped single walled zigzag $(5,0)$ BNT. The Fermi energy is set corresponding to zero energy and represented by dotted line.

point $\mathrm{G}$ is observed to be $1.19 \mathrm{eV}$ but $0.59 \mathrm{eV}$ at another symmetric point $Z$. Despite symmetric points, the band gap $0.38 \mathrm{eV}$ is noticed somewhere between $\mathrm{G}$ and Z. The DOS of the concerned tube also defenses the semiconducting nature as evident from Figure 10.

Thus, the doping of $\mathrm{N}$ in this convolution has remarkable properties and is found to be semiconducting in contrast to metallic one, as observed in pristine form. This transformation may be caused due to the charge transfer from boron to $\mathrm{N}$ atoms. As the concentration increases, more charge transfer takes place but the difference observed in the band gap is meagre. It is also evident from Table 1 that zigzag $(5,0)$ BNT and CNT are metallic while BNNT and SiCNT are semiconducting. In our study, it is found that the $\mathrm{N}$-doped zigzag $(5,0)$ BNT has the band gap lying between SiCNT and BNNT. Thus, these remarkable properties are very suitable for nanooptoelectronic devices. 


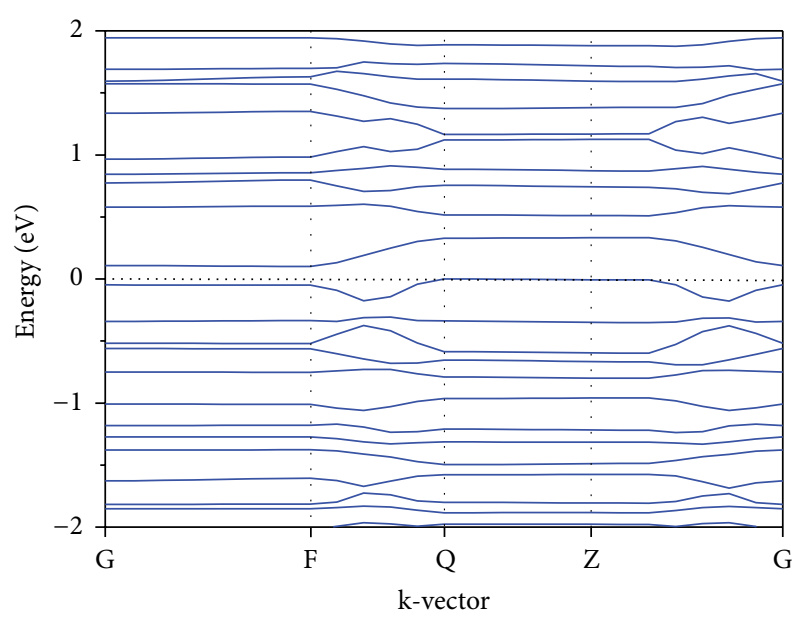

FIGURE 11: The electronic band structure of N1-doped single walled chiral $(4,2)$ BNT. The Fermi level is set corresponding to zero energy and represented by dotted line.

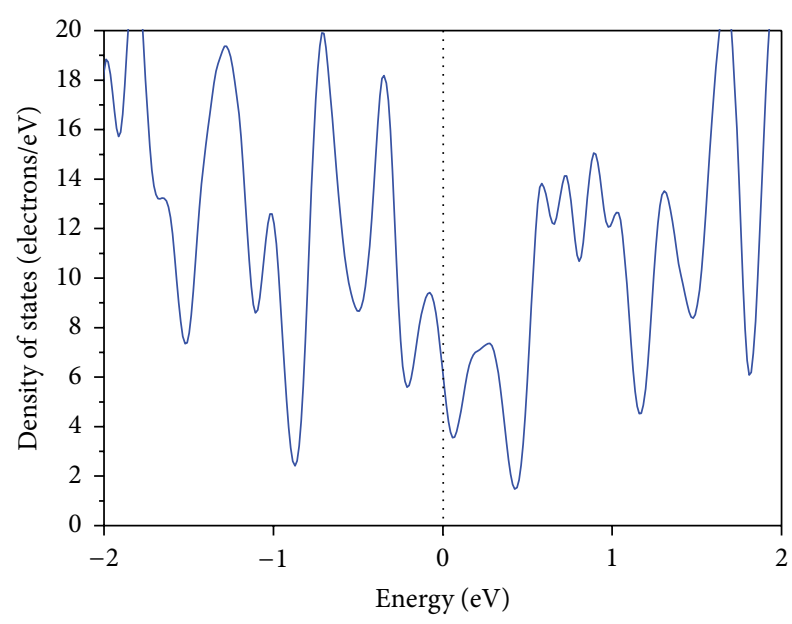

FIGURE 12: Density of states (DOS) of N1-doped single walled chiral $(4,2)$ BNT. The Fermi energy is set corresponding to zero energy and represented by dotted line.

3.3. Chiral $(4,2)$ Boron Nanotube. The electronic band structure of N1-doped chiral $(4,2)$ BNT is shown in Figure 11 and found quite different from achiral BNTs. Here, the energy bands are flat that correspond to large effective mass and high conductivity. None of the energy states cross the Fermi level and have explicit band gap of $0.16 \mathrm{eV}$ at central symmetry point $(\mathrm{G})$. The band gap at $\mathrm{Z}$ is $0.34 \mathrm{eV}$ but $0.101 \mathrm{eV}$ somewhere between symmetric points $\mathrm{G}$ and $\mathrm{Z}$. In contrast, the band gap $0.43 \mathrm{eV}$ was observed for pristine chiral $(4,2)$ BNT. After introduction of impurity, the band gap is reduced which is attributed to extra electrons of $\mathrm{N}$ impurity. The extra electrons of $\mathrm{N}$ impurity provide additional electronic states in the vicinity of Fermi level causing reduction in the band gap. Thus, the semiconducting tube transformed into the semimetallic tube. The concerned DOS is depicted in Figure 12 showing high DOS in the vicinity of Fermi level and

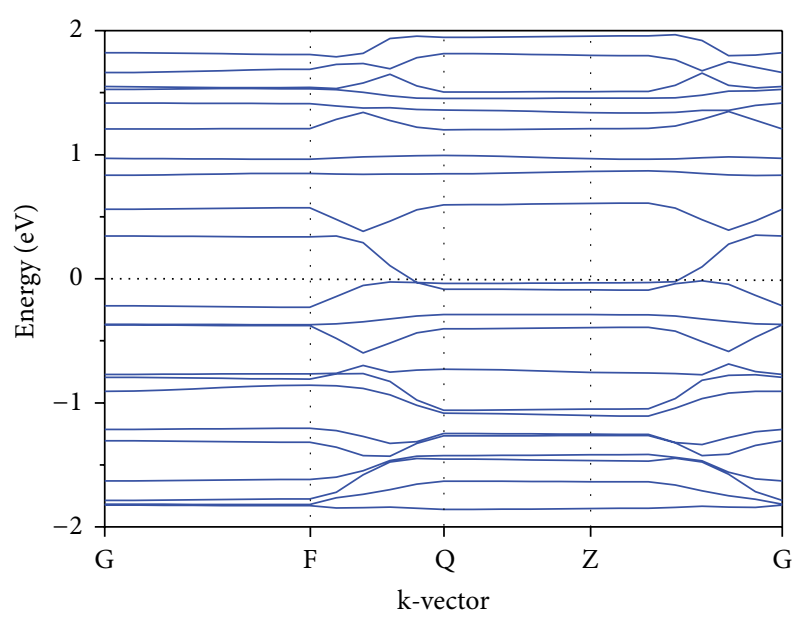

FIGURE 13: The electronic band structure of N2-doped single walled chiral $(4,2)$ BNT. The Fermi level is set corresponding to zero energy and represented by dotted line.

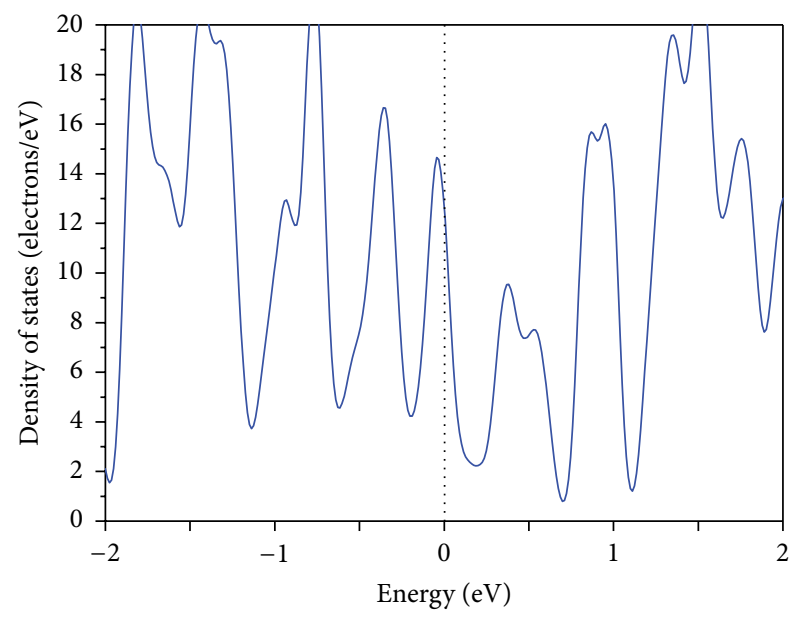

FIGURE 14: Density of states (DOS) of N2-doped single walled chiral $(4,2)$ BNT. The Fermi energy is set corresponding to zero energy and represented by dotted line.

the low magnitude states crossing the Fermi level confirm the semimetallic nature.

Again if we increase the concentration of $\mathrm{N}$ atoms to two, the energy states become more and more close to Fermi level and finally intersect it as shown in Figure 13. The nitrogen atoms are connected to three atoms of boron and transfer charge to $\mathrm{N}$ atoms which gives rise to high conductivity. Here, the bands are also flat, indicating large effective mass results in high conductivity. The corresponding DOS is shown in Figure 14, and one can observe high DOS at Fermi level. Here, the DOS is shifted towards high energy side in comparison to one $\mathrm{N}$-atom doping.

Thus, nitrogen doping in BNTs enhances both stability [34] and conductivity in comparison to pristine ones. However, nitrogen doping in CNT gives high conductivity and low stability in contrast to pristine ones [30-33]. Moreover, it is also noteworthy here that N-doped chiral $(4,2)$ BNT is 
found to be semimetallic. In contrast, CNT, BNNT, SiCNT, and pristine BNT all are found to be semiconducting. The band gap of N-doped BNT is smaller than those of BNNT and SiCNT which suggest its applications for small band gap semiconducting devices.

\section{Conclusion}

In summary, we performed first principles calculations for exploring the effect of nitrogen impurity on the electronic properties of boron nanotubes of diameter below $5 \AA$. It is found that the nitrogen impurity $(\mathrm{N})$ enhances the conductivity of armchair $(3,3)$ BNT. Therefore, the $(3,3)$ BNTs are the most desirable candidate for field emission devices and metallic contacts. In contrast, the $(5,0)$ BNT doped with nitrogen is observed to be semiconducting. Further, the chiral $(4,2)$ BNT which is semiconducting in its pristine form turns into narrow band gap semiconductor or semimetals after nitrogen doping. Thus, the nitrogen impurity reduces the band gap in chiral BNT. Consequently, nitrogen doping has significant effect on chiral and achiral nanotubes. Experimental workers are warranted to verify our findings.

\section{Conflict of Interests}

The authors declare that there is no conflict of interests regarding the publication of this paper.

\section{Acknowledgment}

The authors are thankful to Computational Nanoscience \& Technology Lab, ABV-Indian Institute of Information Technology \& Management, Gwalior (MP), India, for providing computational facility.

\section{References}

[1] S. Iijima, "Helical microtubules of graphitic carbon," Nature, vol. 354, no. 6348, pp. 56-58, 1991.

[2] R. Saito and G. Dresselhaus, Physical Properties of Carbon Nanotubes, Imperial College Press, London, UK, 1998.

[3] R. Saito, M. Fujita, G. Dresselhaus, and M. S. Dresselhaus, "Electronic structure of chiral graphene tubules," Applied Physics Letters, vol. 60, no. 18, pp. 2204-2206, 1992.

[4] N. Hamada, S.-I. Sawada, and A. Oshiyama, "New onedimensional conductors: graphitic microtubules," Physical Review Letters, vol. 68, no. 10, pp. 1579-1581, 1992.

[5] R. Heyd, A. Charlier, and E. McRae, "Uniaxial-stress effects on the electronic properties of carbon nanotubes," Physical Review $B$, vol. 55, no. 11, pp. 6820-6824, 1997.

[6] T. W. Tombler, C. Zhou, L. Alexseyev et al., "Reversible electromechanical characteristics of carbon nanotubes under localprobe manipulation," Nature, vol. 405, no. 6788, pp. 769-772, 2000.

[7] L. Yang and J. Han, "Electronic structure of deformed carbon nanotubes," Physical Review Letters, vol. 85, no. 1, pp. 154-157, 2000 .
[8] J. Lee, H. Kim, S.-J. Kahng et al., "Bandgap modulation of carbon nanotubes by encapsulated metallofullerenes," Nature, vol. 415, no. 6875, pp. 1005-1008, 2002.

[9] S. J. Tans, A. R. M. Verschueren, and C. Dekker, "Roomtemperature transistor based on a single carbon nanotube," Nature, vol. 393, no. 6680, pp. 49-52, 1998.

[10] W. A. De Heer, A. Châtelain, and D. Ugarte, "A carbon nanotube field-emission electron source," Science, vol. 270, no. 5239, pp. 1179-1180, 1995.

[11] I. Boustani and A. Quandt, "Nanotubules of bare boron clusters: ab initio and density functional study," Europhysics Letters, vol. 39, no. 5, pp. 527-532, 1997.

[12] I. Boustani, A. Quandt, E. Hernández, and A. Rubio, "New boron based nanostructured materials," Journal of Chemical Physics, vol. 110, no. 2-12, pp. 3176-3185, 1999.

[13] D. Ciuparu, R. F. Klie, Y. Zhu, and L. Pfefferle, "Synthesis of pure boron single-wall nanotubes," Journal of Physical Chemistry B, vol. 108, no. 13, pp. 3967-3969, 2004.

[14] K. C. Lau and R. Pandey, "Thermodynamic stability of novel boron sheet configurations," Journal of Physical Chemistry B, vol. 112, no. 33, pp. 10217-10220, 2008.

[15] A. K. Singh, A. Sadrzadeh, and B. I. Yakobson, "Probing properties of boron a-tubes by ab initio calculations," Nano Letters, vol. 8, no. 5, pp. 1314-1317, 2008.

[16] X. Yang, Y. Ding, and J. Ni, "Ab initio prediction of stable boron sheets and boron nanotubes: structure, stability, and electronic properties," Physical Review B, vol. 77, no. 4, Article ID 041402, 2008.

[17] Y. Ding and J. Ni, "Electronic properties of boron nanotubes with axial strain," Frontiers of Physics in China, vol. 4, no. 3, pp. 383-388, 2009

[18] J. Kunstmann and A. Quandt, "Broad boron sheets and boron nanotubes: an ab initio study of structural, electronic, and mechanical properties," Physical Review B, vol. 74, no. 3, Article ID 035413, 2006.

[19] D. Zhang, R. Zhu, and C. Liu, "Density functional theory study on the geometrical and electronic structures of a new thinnest boron nanotube," Journal of Materials Chemistry, vol. 16, no. 25, pp. 2429-2433, 2006.

[20] S. K. Jain and P. Srivastava, "Electronic and optical properties of ultrathin single walled boron nanotubes-an ab initio study," Computational Materials Science, vol. 50, no. 10, pp. 3038-3042, 2011.

[21] F. Liu, C. Shen, Z. Su et al., "Metal-like single crystalline boron nanotubes: synthesis and in situ study on electric transport and field emission properties," Journal of Materials Chemistry, vol. 20, no. 11, pp. 2197-2205, 2010.

[22] K. C. Lau, R. Pandey, R. Pati, and S. P. Karna, "Theoretical study of electron transport in boron nanotubes," Applied Physics Letters, vol. 88, no. 21, Article ID 212111, 2006.

[23] V. Bezugly, J. Kunstmann, B. Grundkötter-Stock, T. Frauenheim, T. Niehaus, and G. Cuniberti, "Highly conductive boron nanotubes: transport properties, work functions, and structural stabilities," ACS Nano, vol. 5, no. 6, pp. 4997-5005, 2011.

[24] M. Glerup, J. Steinmetz, D. Samaille et al., "Synthesis of Ndoped SWNT using the arc-discharge procedure," Chemical Physics Letters, vol. 387, no. 1-3, pp. 193-197, 2004.

[25] L. Duclaux, "Review of the doping of carbon nanotubes (multiwalled and single-walled)," Carbon, vol. 40, no. 10, pp. 1751-1764, 2002. 
[26] M. Terrones, A. Jorio, M. Endo et al., "New direction in nanotube science," Materials Today, vol. 7, no. 9, pp. 30-45, 2004.

[27] C. P. Ewels and M. Glerup, "Nitrogen doping in carbon nanotubes," Journal of Nanoscience and Nanotechnology, vol. 5, no. 9, pp. 1345-1363, 2005.

[28] F. Villalpando-Paez, A. Zamudio, A. L. Elias et al., "Synthesis and characterization of long strands of nitrogen-doped singlewalled carbon nanotubes," Chemical Physics Letters, vol. 424, no. 4-6, pp. 345-352, 2006.

[29] Y.-S. Min, E. J. Bae, I. P. Asanov, U. J. Kim, and W. Park, "Growth and characterization of nitrogen-doped single-walled carbon nanotubes by water-plasma chemical vapour deposition," Nanotechnology, vol. 18, no. 28, Article ID 285601, 2007.

[30] T. Loponen, A. V. Krasheninnikov, M. Kaukonen, and R. M. Nieminen, "Nitrogen-doped carbon nanotubes under electron irradiation simulated with a tight-binding model," Physical Review B, vol. 74, no. 7, Article ID 073409, 2006.

[31] G. Zhang, W. H. Duan, and B. L. Gu, "Effect of substitutional atoms in the tip on field-emission properties of capped carbon nanotubes," Applied Physics Letters, vol. 80, pp. 2589-2591, 2002.

[32] G. Kresse and D. Joubert, "From ultrasoft pseudopotentials to the projector augmented-wave method," Physical Review B, vol. 59, no. 3, pp. 1758-1775, 1999.

[33] H.-L. Park, S.-C. Yi, and Y.-C. Chung, "Electronic properties of nitrogen-doped carbon nanotubes with strain: ab initio method approach," Japanese Journal of Applied Physics, vol. 47, no. 6, pp. 5062-5065, 2008.

[34] S. K. Jain and P. Srivastava, "Structural stability of nitrogendoped ultrathin single-walled boron nanotubes: an ab initio study," Applied Nanoscience, vol. 2, no. 3, pp. 345-349, 2012.

[35] S. J. Clark, M. D. Segall, C. J. Pickard et al., "First principles methods using CASTEP," Zeitschrift fur Kristallographie, vol. 220, no. 5-6, pp. 567-570, 2005.

[36] J. P. Perdew, K. Burke, and M. Ernzerhof, "Generalized gradient approximation made simple," Physical Review Letters, vol. 77, no. 18, pp. 3865-3868, 1996.

[37] H. J. Monkhorst and J. D. Pack, "Special points for Brillouinzone integrations," Physical Review B, vol. 13, no. 12, pp. 51885192, 1976.

[38] D. Vanderbilt, "Soft self-consistent pseudopotentials in a generalized eigenvalue formalism," Physical Review B, vol. 41, no. 11, pp. 7892-7895, 1990.

[39] G. Y. Guo, K. C. Chu, D.-S. Wang, and C.-G. Duan, "Linear and nonlinear optical properties of carbon nanotubes from first-principles calculations," Physical Review B, vol. 69, no. 20, Article ID 205416, 2004.

[40] I. J. Wu and G. Y. Guo, "Optical properties of SiC nanotubes: an ab initio study," Physical Review B, vol. 76, no. 3, Article ID 035343, 9 pages, 2007.

[41] Y. L. Wang, J. P. Zhang, K. H. Su, X. Wang, Y. Liu, and X. Sun, "A density functional theory study on parameters fitting of ultra long armchair (n, n) single walled boron nitride nanotubes," Chinese Physics B, vol. 21, no. 6, Article ID 060301, 2012.

[42] G. Y. Guo and J. C. Lin, "Systematic ab initio study of the optical properties of BN nanotubes," Physical Review B, vol. 71, no. 16, Article ID 165402, 12 pages, 2005.

[43] H. Tang and S. Ismail-Beigi, "Novel precursors for boron nanotubes: the competition of two-center and three-center bonding in boron sheets," Physical Review Letters, vol. 99, no. 11, Article ID 115501, 2007. 

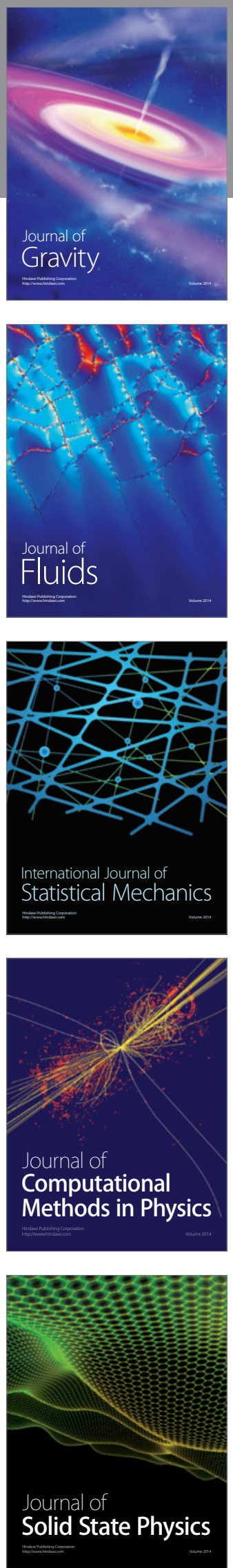

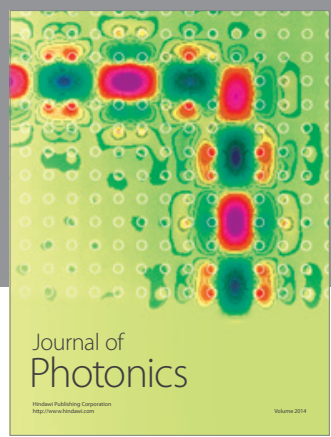

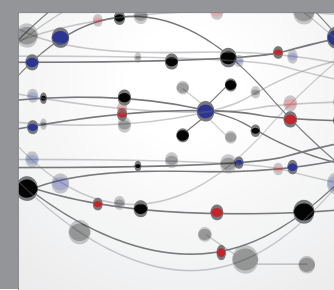

The Scientific World Journal

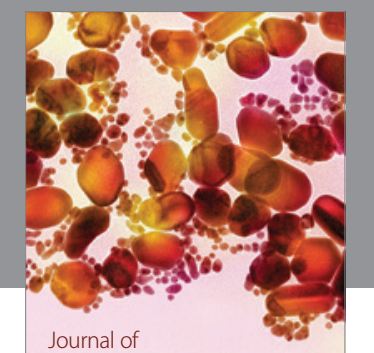

Soft Matter
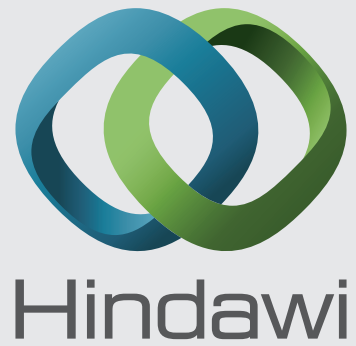

Submit your manuscripts at

http://www.hindawi.com
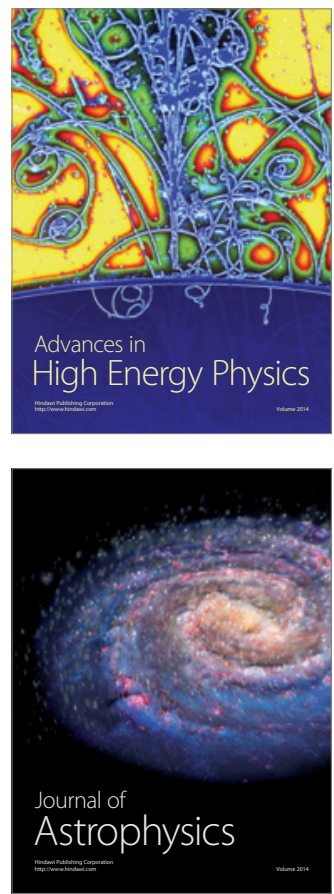
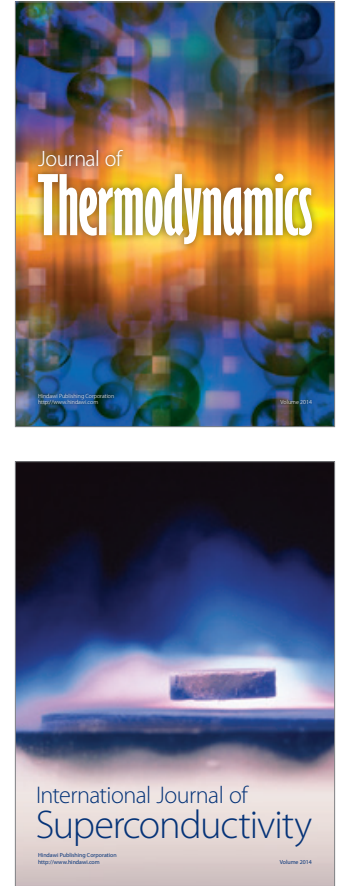
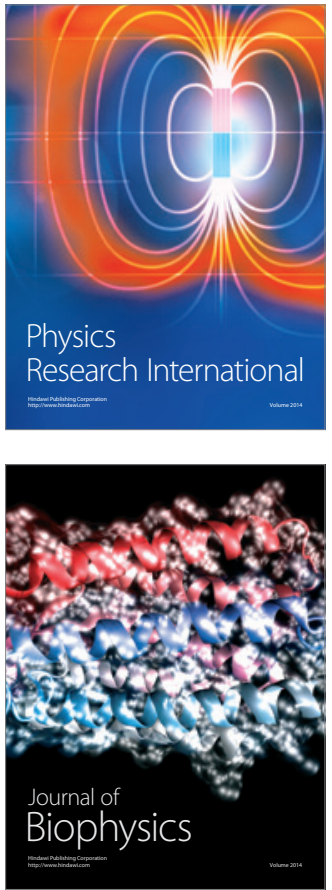
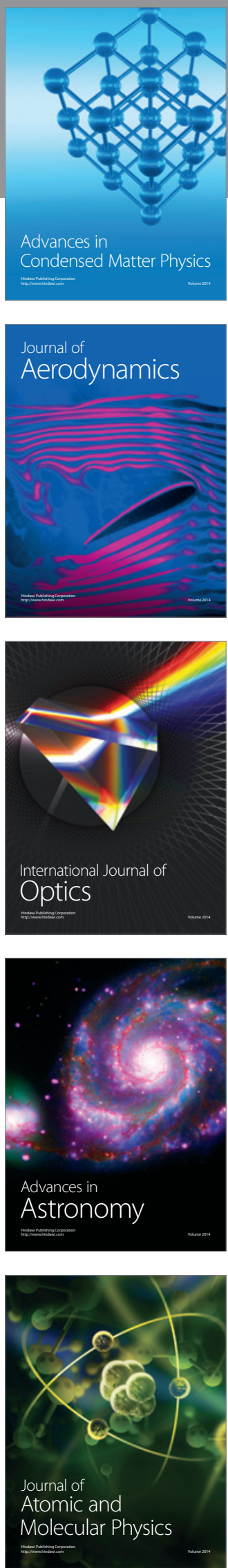